\title{
Respiratory Arrest Induced by Presbyesophagus: A Case Report
}

\author{
Yong Sung Jeong, M.D., InHyuk Suh, M.D., Jong Keun Kim, M.D., Jin Seok Bae, M.D., \\ Jong Youb Lim, M.D., Ph.D. \\ Department of Rehabilitation Medicine, Daejeon Eulji University Hospital, Eulji University School of Medicine, \\ Daejeon, Korea
}

\begin{abstract}
Presbyesophagus is a unique cause of dysphagia in the elderly, which features incomplete relaxation of the lower esophageal sphincter, dilatation of the esophagus, decreased esophageal peristaltic pressures, abnormal esophageal contractions, and delayed emptying of the esophagus. An 88-year-old woman underwent surgery for a femur fracture. The day after surgery, respiratory arrest occurred during a meal. Breathing resumed after oxygen supply with airway opening. She complained of dysphagia and nausea during a meal, and the videofluoroscopic swallowing study and esophagography showed delayed emptying of the esophagus, abnormal esophageal contraction, and dilatation of the esophagus. This case shows that esophageal dysfunction in the elderly with poor general medical conditions can worsen abruptly. (JKDS 2021;11:78-81)
\end{abstract}

Keywords: Presbyesophagus, Respiratory arrest, Videofluoroscopic swallowing study, Esophagography

\section{INTRODUCTION}

Aging is a bodily change that occurs in everyone over time, and the functional impairment of the organs due to the progression of physiological deterioration is one important change associated with aging ${ }^{1}$. Presbyesophagus is the term used to describe the age-related changes in the esophagus, including incomplete relaxation of the lower esophageal sphincter, dilatation of the esophagus, decreased esophageal peristaltic pressures, abnormal esophageal contractions, and delayed emptying of the esophagus ${ }^{2}$. Adverse effects of aging also contribute to the increased sensory threshold and atypical reflux symp- toms $^{3}$. Poor general medical conditions also can lead to a marked decline in digestion ${ }^{1}$.

When these situations overlap, they can lead to a severe incident. Here we report a case of respiratory arrest of an 88-year-old woman after a femur fracture operation, the cause of which was later diagnosed as presbyesophagus by conducting a videofluoroscopic swallowing study (VFSS), endoscopy, and esophagography.

\section{CASE REPORT}

An 88-year-old woman visited the emergency department. She had slipped and fallen at home, and
Received: June 12 2020, Revised: June 16 2020,

Accepted: July 62020

Corresponding author: Jong Youb Lim, Department of Rehabilitation Medicine, Daejeon Eulji University Hospital, Eulji University School of Medicine, 95 Dunsanseo-ro, Seo-gu, Daejeon 35233, Korea Tel: +82-42-611-3631, Fax: +82-42-611-3633 E-mail: jylimmd@eulji.ac.kr
Copyrights (c) The Korean Dysphagia Society, 2021. 
a femur fracture was diagnosed. She was admitted to the department of orthopedic surgery and received an operation. She had no special complications related to the operation on that day. The day after the surgery, she started eating a regular meal. During a meal, she showed cyanosis. A pulse was taken, but she was not breathing. Immediately the airway was opened, and oxygen was supplied. After that, breathing resumed and she was transferred to the intensive care unit. After she regained consciousness, she complained of dysphagia and nausea during a meal. She said she had intermittent chest discomfort and vomiting after meals before hospitalization.

No specific substance which could have blocked the airway was found in the hospital meal. She denied any change in phonation. Also, she had no symptoms such as dyspnea, heartburn and abdominal pain. She only took painkiller medication. She did not take opioids, sedative-hypnotics or alcohol, all of which can decrease respiratory effort. There was no significant psychiatric history or family history.

Physical examination showed an alert, fully oriented, non-cachectic female with normal skin. On auscultation, the lung sound was clear and no abnormal sound such as rale, crackle, or wheezing was found. Abdominal examination showed no significant findings. Neurological examination also did not show any focal neurological deficit, and the cranial nerve examination was within normal limits. Laboratory examin- ation showed white blood cells at $10,920 / \mathrm{mm}^{3}$ (normal range $3,800-10,500 / \mathrm{mm}^{3}$ ) and C-reactive protein at $4.9 \mathrm{mg} / \mathrm{dL}$ (normal range $0-0.5 \mathrm{mg} / \mathrm{dL}$ ). Inflammatory marker levels were elevated after surgery, and the levels were not high enough to cause sepsis. Additionally, the change between the previous and followup tests was small. A chest simple radiograph indicated calcified granuloma in the right upper lung field, but this finding was consistent with previous results. On chest computed tomography, diffuse luminal dilatation of the esophagus without definite obstruction was found. However, obstruction of the airway, as well as pneumonia, pulmonary edema, and pulmonary hemorrhage were not observed. The brain and heart exploration also showed no structural abnormalities on computed tomography and no specific findings on echocardiogram.

In the symptoms and examinations so far, no definite cause for the respiratory arrest was found, and further evaluations were performed. Upon VFSS, there was no evidence of aspiration or penetration in the airway, nor retention within the valleculae or pyriform sinuses. Additionally, there were no significant findings on the oral and pharyngeal phases. However, on the esophageal phase, delayed emptying of the esophagus, abnormal esophageal contraction, and dilatation of the esophagus were found.(Fig. 1A) Also, these findings were found to be more severe in the regular diet than in the thick-liquid diet and
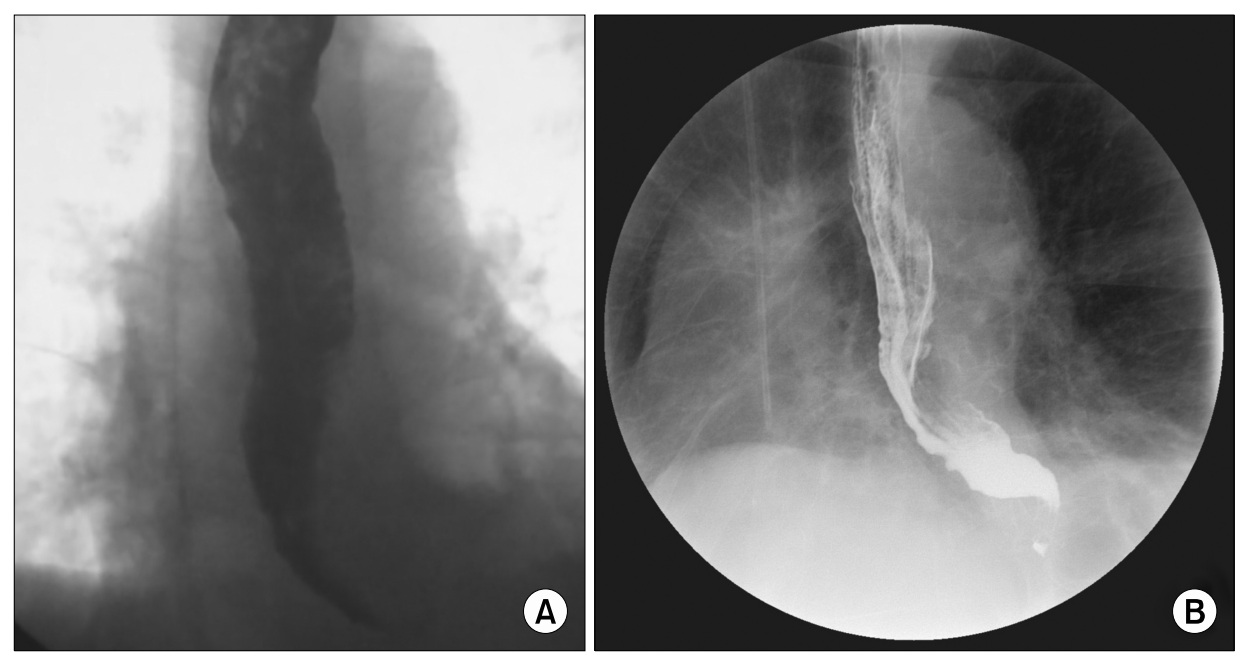

Fig. 1. Presbyesophagus diagnosed by VFSS and esophagography. (A) VFSS showed delayed emptying of the esophagus. (B) Esophagography showed delayed relaxation of the lower esophageal sphincter. 
soft-blended diet. Endoscopy showed dilatation of the esophagus, but the lower esophageal tone was not increased. On esophagography, the esophagus did not indicate abnormal filling defects or barium collections. Additionally, there were no abnormal findings at the esophagogastric junction. However, delayed relaxation of the lower esophageal sphincter was found.(Fig. 1B)

Presbyesophagus was confirmed, and the patient was recommended to start with a combination of liquid diet and dyspepsia medicine. Gradually the diet was altered with a small amount of puree diet, and she was encouraged to eat slowly. VFSS performed three weeks after diet modification and medicine showed improvement, and the patient was able to swallow a regular diet.

\section{DISCUSSION}

Presbyesophagus is the change in the structure of the esophagus which causes an alteration in esophageal motility. It is characterized by abnormal contractions and incomplete relaxations of the upper and lower esophageal sphincters, as well as esophageal dilatation ${ }^{2}$.

Older people have gastrointestinal diseases more frequently ${ }^{1}$. Among them, presbyesophagus is more difficult to diagnose, because it appears in the very elderly and does not present with prominent symptoms such as abdominal pain, odynophagia, and weight loss. In this case, respiratory arrest occurred while eating, so the possibility of airway obstruction due to certain foods was highly suspected. However, there was no specific substance in her hospital meal that could cause obstruction. Additional tests performed to rule out common causes of respiratory arrest found no definite explanation for this patient.

As a further means of examination, VFSS was done. We initially focused on the pharyngeal phase to look for any aspiration. But there was no evidence of aspiration or retention within the valleculae or pyriform sinuses. To evaluate the esophageal phase, we fed the patient a liquid diet, thick-liquid diet, soft- blended diet, and regular diet in order, with the portion amount being half of a paper cup, respectively. However, only a small amount of food was moved to the patient's stomach, while most of the meals were found to remain in the esophagus. And these findings were the most prominent in the regular diet. Esophageal emptying is highly correlated with bolus viscosity ${ }^{4}$. Diet volume and bolus viscosity affect esophageal function ${ }^{5}$. Therefore, it was suggested that there was a possibility of respiratory arrest, due to the decrease of esophageal peristalsis when the whole hospital meal was fed to the patient.

Dilatation of the esophagus and abnormal esophageal contraction can be caused by a variety of diseases, when considering the integrated pressures of relaxation and the movement of peristalsis ${ }^{6}$. Achalasia can be identified by high integrated pressures of relaxation; however, the pressure measurement by endoscopy in this patient showed no such findings. On esophagography, there were decreased frequencies of normal peristalsis and tertiary contractions suggestive of presbyesophagus ${ }^{2}$. Diffuse esophageal spasm and obstruction effect were not found. No mass was observed to press on the esophagus in the chest computed tomography. Taking all these findings into account, it was concluded that the abnormal esophageal peristalsis was caused by presbyesophagus.

Presbyesophagus is a disease particularly affecting the elderly, as it occurs due to normal aging. Therefore, individuals above 80 years of age are more likely to develop presbyesophagus ${ }^{7}$. Esophageal peristalsis is more likely to fail in patients with a systemic illness or poor general medical conditions. In this case, the patient was 88 years old, a very elderly woman, and she had received an operation the day before the respiratory arrest. VFSS and esophagography were performed about 10 days after the respiratory arrest, but the presbyesophagus might have been at its worst on the day of arrest.

The mechanism of respiratory arrest in the present patient was probably obstructive. In the patient we have reported, the esophagus was dilated to the upper and middle esophagus on VFSS due to decreased 
peristalsis. There were several cases of airway obstruction caused by the expansion of the upper esophagus $^{8-10}$. Also, there was a hypothesis that an irregularly expanded esophagus creates a 1-way valve where air flows only on one side, compressing the upper airway ${ }^{11}$.

This case shows that dysphagia due to presbyesophagus might become more severe after events such as surgery that could worsen general medical conditions. In treating any aged people with poor general medical conditions, we should pay more attention to dysphagia and start a small amount of diet with diet modification and medications. Additionally, if the patient complains of a symptom consistent with dysphagia, further evaluation is warranted.

\section{CONFLICT OF INTEREST}

The authors declare that they have no conflict of interest.

\section{REFERENCES}

1. Dumic I, Nordin T, Jecmenica M, Stojkovic Lalosevic M, Milosavljevic T, Milovanovic T. Gastrointestinal tract disorders in older age. Can J Gastroenterol Hepatol. 2019;
2019:6757524.

2. DeVault KR. Presbyesophagus: a reappraisal. Curr Gastroenterol Rep. 2002;4:193-9.

3. Cock C, Besanko LK, Burgstad CM, Thompson A, Kritas $\mathrm{S}$, Heddle R, et al. Age-related impairment of esophagogastric junction relaxation and bolus flow time. World $\mathrm{J}$ Gastroenterol. 2017;23:2785-94.

4. Kim CH, Hsu JJ, O'Connor MK, Weaver AL, Brown ML, Zinsmeister AR. Effect of viscosity on oropharyngeal and esophageal emptying in man. Dig Dis Sci. 1994;39:18992.

5. Omari TI, Wauters L, Rommel N, Kritas S, Myers JC. Oesophageal pressure-flow metrics in relation to bolus volume, bolus consistency, and bolus perception. United European Gastroenterol J. 2013;1:249-58.

6. Shenoda B, Degen KC, Ford W. Presbyesophagus presented with chronic intermittent dysphagia. Aging Clin Exp Res. 2019;31:1343-6.

7. Dantas RO, Alves LM, Dalmazo J, Santos CM, Cassiani Rde A, Nascimento WV. Effect of age on proximal esophageal response to swallowing. Arq Gastroenterol. 2010; 47:339-43.

8. Dunlop SP, Travis SP. Achalasia presenting as acute stridor. Eur J Gastroenterol Hepatol. 1997;9:1125-8.

9. Arcos E, Medina C, Mearin F, Larish J, Guarner L, Malagelada JR. Achalasia presenting as acute airway obstruction. Dig Dis Sci. 2000;45:2079-83.

10. Moloney JR, Carr-Locke DL, Cookson JB. Stridor due to an achalasia-like condition of the oesophagus. J Laryngol Otol. 1987;101:1297-300.

11. Wagh MS, Matloff DS, Carr-Locke DL. Life-threatening acute airway obstruction in achalasia. MedGenMed. 2004; $6: 12$ 